Article

\title{
Interface Effects on Screw Dislocations in Heterostructures
}

\author{
Jianwei Wang ${ }^{1}$, Ting Sun ${ }^{2}$, Weiwei $\mathrm{Xu}^{2}{ }^{2}$, Xiaozhi Wu ${ }^{2,3, *}$ and Rui Wang ${ }^{2}$ \\ 1 Microsystem and Terahertz Research Center, CAEP, Chengdu 610200, China; wangjianwei@mtrc.ac.cn \\ 2 Institute for Structure and Function, Chongqing University, Chongqing 401331, China; \\ sunting@cqu.edu.cn (T.S.); xuweiwei0308@cqu.edu.cn (W.X.); rcwang@cqu.edu.cn (R.W.) \\ 3 College of Materials Science and Engineering, Chongqing University, Chongqing 400044, China \\ * Correspondence: xiaozhiwu@cqu.edu.cn
}

Received: 14 November 2017; Accepted: 8 January 2018; Published: 10 January 2018

\begin{abstract}
The governing equation of screw dislocations in heterostructures is constructed using image method. The interface type $(-1 \leq \gamma \leq 1)$ and distance between dislocation and interface $h$ are considered in the new equation. The Peierls-Nabarro equations for screw dislocations in bulk and semi-infinite materials can be recovered when $\gamma=0$ and $\gamma=-1$. The soft $(\gamma<0)$ and hard $(\gamma>0)$ interfaces can enhance and reduce the Peierls stress of screw dislocations near the interface, respectively. The interface effects on dislocations decrease with the increasing of distance $h$. The $\mathrm{Al} / \mathrm{TiC}$ heterostructure is investigated as a model interface to study the unstable stacking fault energy and dislocation properties of the interface. The mismatch of lattice constants and shear modulus at the interface results in changes of the unstable stacking fault energy. Then, the changes of the unstable stacking fault energy also have an important effect on dislocation properties, comparing with $\gamma$ and $h$.
\end{abstract}

Keywords: interface effects; screw dislocation; image method; Peierls-Nabarro model

\section{Introduction}

Dislocations play an important role in understanding the mechanical properties of crystalline solids [1]. The continuum elasticity theory can describe the long-range elastic field of dislocations well, but it fails in dislocation core region due to the singularity. The influence of the lattice periodicity of real crystals on dislocation properties should be taken into account. The classical Peierls-Nabarro (P-N) model provides a useful approach to determining the core properties of dislocations [2,3]. Based on the finite scale of the dislocation core, we can estimate the minimum external stress required to move a dislocation in a perfect crystal-the so-called Peierls stress. The predictions can be improved when the sinusoidial force law is replaced by the generalized stacking fault (GSF) energy [4-6]. Then, the P-N model combined GSF energy is extensively and successfully used to predict the dislocation properties in bulk materials $[7,8]$.

It is widely accepted that the dislocation core and mobility can be influenced by various inhomogeneities, such as surface, interfaces, and cavities in materials [9]. Lee and Li yielded a half-space P-N model for dislocations near a free surface in semi-infinite materials with single surface $[10,11]$. Cheng et al. constructed the semidiscrete P-N model to characterize the surface on dislocations using the image dislocation method [12]. The Peierls stress is increased when dislocations are located near the free surface. The results are in agreement with the molecular dynamics simulation. The heterostructures and laminated structures are very common in modern science for the design of strong structural materials. Within continuum elasticity theory, Head investigated the interaction of screw dislocations and interfaces in heterostructures using the image method [13]. When the screw dislocation is located in a rigid (soft) material, the dislocations are attracted (repelled) by the interface. 
The attraction and repulsion between dislocation and interface are only affected by the mismatch in the shear modulus of two semi-infinite materials. However, two other sources also arise in the interaction of dislocations and interface. One is the mis-fit of lattice constants at the interface. This will change the atomic arrangement near the interface. Then, the GSF energy near the interface will be altered. The other is the distance between the interface and dislocations. When the distance is large enough, the atomic arrangement on GSF energy is small due to the locality of GSF energy. Only the elastic interaction is expected.

In this paper, the interface effects on screw dislocation are investigated using the image method in heterostructures. The image dislocations are introduced to satisfy the interface boundary condition. The stress and displacement should be continuous across the interface. The governing equation for screw dislocations is presented according to the procedure for the P-N equation in an elegant manner. The mismatch of shear modulus, mis-fit of lattice constants, and the distance between interface and dislocations on the dislocation core and Peierls stress are considered. Furthermore, the interface effects on screw dislocations in $\mathrm{Al} / \mathrm{TiC}$ heterostructure are also presented.

\section{Screw Dislocation Equation with Interfacial Effects}

In order to take the interfacial effects on screw dislocations into account, an original right-handed screw dislocation parallel to the interface is represented by $\mathrm{O}$ (see Figure 1). In a cartesian set of coordinates $x y z$, the original dislocation line is set to along the $z$ axis, the $x o z$ plane is the slip plane, and $y$ is normal to the slip plane. The $y=h$ plane is the interface of material-I and material-II. Material-I and material-II are placed at $y<h$ and $y>h$. The shear moduli of material-I and material-II are $\mu_{I}$ and $\mu_{I I}$, respectively. Therefore, $h$ is the distance between interface and dislocation. Due to the equivalence of dislocations and point charges in elastic strain field and electrostatics, the image method is used to describe the interface effects on the dislocation elastic strain field. The interface effects on dislocations are represented by image dislocations. The image dislocation of $\mathrm{O}$ is at $\mathrm{A}$. As we know, there is no strain field in material-II while the interface is not taken into account. The interface between material-I and material-II will introduce a strain field in the material. Accordingly, the interface effects on dislocation $\mathrm{O}$ is represented by image dislocation $\mathrm{A}$. To account for finite strain in material-II, an additional image is placed at $\mathrm{B}$ to describe the interface effects. The dislocations $\mathrm{O}$, $A$, and $B$ have Burgers vectors of magnitudes $b, \gamma b$, and $\beta b$, respectively. According to the required boundary conditions that the displacements and stress must be continuous across the interface, the three dislocations must lie in the plane $x=0$, and in fact, the results verify that such an arrangement fulfills the boundary conditions. Additionally, in order for the displacements at the interface to vary in the same way with varying $x$, it is necessary that $L_{O}=L_{A}=L_{B}$. The displacement and stress of the original screw dislocation are

$$
\begin{aligned}
u_{z} & =\frac{b}{2 \pi} \arctan \frac{y}{x} \\
\sigma_{y z} & =\frac{\mu b}{2 \pi} \frac{x}{x^{2}+y^{2}} .
\end{aligned}
$$

It is important to elucidate that both image dislocations A and B are the images of the original screw dislocation O. In other words, the strain field in material-I is the same as that which would be produced by dislocation $\mathrm{O}$ and the image dislocation $\mathrm{A}$, while the strain field in material-II is the same as that produced by dislocation B. Therefore, the displacement in material-I and material-II can be written as

$$
\begin{aligned}
u_{z_{I}} & =\frac{b}{2 \pi}\left(\arctan \frac{y}{x}+\gamma \arctan \frac{y-2 h}{x}\right) \\
u_{z_{I I}} & =\frac{\beta b}{2 \pi} \arctan \frac{y}{x}
\end{aligned}
$$


and the stress in material-I and material-II can be written as

$$
\begin{aligned}
\sigma_{y z_{I}} & =\frac{\mu_{I} b x}{2 \pi\left(x^{2}+y^{2}\right)}+\frac{\mu_{I} \gamma b x}{2 \pi\left[\left(x^{2}+(y-2 h)^{2}\right]\right.}, \\
\sigma_{y z_{I I}} & =\frac{\mu_{I I} \beta b}{2 \pi\left(x^{2}+y^{2}\right)} .
\end{aligned}
$$

At the interface $(y=h)$, the displacement and stress must be continuous; namely,

$$
\begin{aligned}
u_{z_{I}} & =u_{z_{I I}} \\
\sigma_{y z_{I}} & =\sigma_{y z_{I I}} .
\end{aligned}
$$

One finds

$$
\begin{aligned}
\beta & =1-\gamma \\
\mu_{I}(1+\gamma) & =\mu_{I I} \beta .
\end{aligned}
$$

It is easy to yield

$$
\begin{aligned}
& \gamma=\frac{\mu_{I I}-\mu_{I}}{\mu_{I}+\mu_{I I}}, \\
& \beta=\frac{2 \mu_{I}}{\mu_{I}+\mu_{I I}} .
\end{aligned}
$$

$\gamma$ can be used to characterize the interface type. Dislocations are in the soft material for $\mu_{I I}>\mu_{I}$ $(1 \geq \gamma>0)$ and the interface can be named as a rigid interface. When $\mu_{I I}<\mu_{I}(-1<\gamma<0)$, dislocations are in a rigid material and the interface can be named as a soft interface. When $\gamma=-1$, the interface is reduced to surface.

Now, the stress field on the slip plane $(y=0)$ with the contribution of image dislocation can be written as

$$
\sigma_{y z}=\frac{\mu_{I} b}{2 \pi}\left[\frac{1}{x}+\gamma \frac{x}{x^{2}+(2 h)^{2}}\right] .
$$

The above discussions are focused on the properties of dislocations in the continuum theory. A dislocation is a singular line in continuum theory, and can be displaced without any application of force. However, the influence of the lattice periodicity on dislocation properties should be taken into account. The P-N model is the first to determine the dislocation width and Peierls stress. In the $\mathrm{P}-\mathrm{N}$ model, the crystal is set to be two semi-infinite crystals. The dislocation is produced from the nontrivial contact of two semi-infinite crystals. The dislocation core is assumed to spread, and the displacements are assumed to be produced by a continuous distribution of infinitesimal dislocations at every point $x^{\prime}$ of Burgers vectors $\rho\left(x^{\prime}\right)=\frac{\mathrm{d} u\left(x^{\prime}\right)}{\mathrm{d} x^{\prime}}$. The total Burgers vector $b=\int_{-\infty}^{+\infty} \rho\left(x^{\prime}\right) \mathrm{d} x^{\prime}$. The infinitesimal dislocations combined with the stress field from elastic theory are used to derive equations for dislocations in bulk and misfit dislocations at the interface $[5,14]$. Accordingly, the governing equation for screw dislocation in heterostructure with the interfacial effects can easily yield

$$
\frac{\mu_{I}}{2 \pi} \int_{-\infty}^{+\infty}\left[\frac{1}{x-x^{\prime}}+\gamma \frac{x-x^{\prime}}{\left(x-x^{\prime}\right)^{2}+(2 h)^{2}}\right] \frac{\mathrm{d} u\left(x^{\prime}\right)}{\mathrm{d} x^{\prime}} \mathrm{d} x^{\prime}=f(u) .
$$

The restoring force $f(u)$ can be obtained from the gradient of GSF energy $\gamma(u)$

$$
\gamma(u)=\frac{\gamma_{u s}}{2}\left(1-\cos \frac{2 \pi u}{b}\right)
$$


with $\gamma_{u s}$ the unstable stacking fault energy [15]. $\gamma_{u s}$ is the maximum energy barrier for a moving dislocation.

Then, we have the restoring force

$$
f(u)=\sigma_{\max } \sin \frac{2 \pi u}{b}
$$

with the maximum restoring stress $\sigma_{\max }=\frac{\pi \gamma_{u s}}{b}$.

It is interesting to discuss the dislocation equation for two limitations:

(i) When $\mu_{2}=\mu_{I}(\gamma=0)$, material-I and material-II are the same material. Both the interface and image dislocation do not exist. One obtains the classical P-N equation for an isolate screw dislocation in bulk:

$$
\frac{\mu}{2 \pi} \int_{-\infty}^{+\infty} \frac{1}{x-x^{\prime}} \frac{\mathrm{d} u\left(x^{\prime}\right)}{\mathrm{d} x^{\prime}} \mathrm{d} x^{\prime}=f(u)
$$

(ii) When $\mu_{2}=0(\gamma=-1)$, material-II is vacuum. The interface reduces to the surface of material-I. One obtains

$$
\frac{\mu}{2 \pi} \int_{-\infty}^{+\infty}\left[\frac{1}{x-x^{\prime}}-\frac{x-x^{\prime}}{\left(x-x^{\prime}\right)^{2}+(2 h)^{2}}\right] \frac{\mathrm{d} u\left(x^{\prime}\right)}{\mathrm{d} x^{\prime}} \mathrm{d} x^{\prime}=f(u)
$$

This equation is the same as the previous dislocation equation obtained by Cheng et al. for a semi-infinite material with seldom free surface [8].

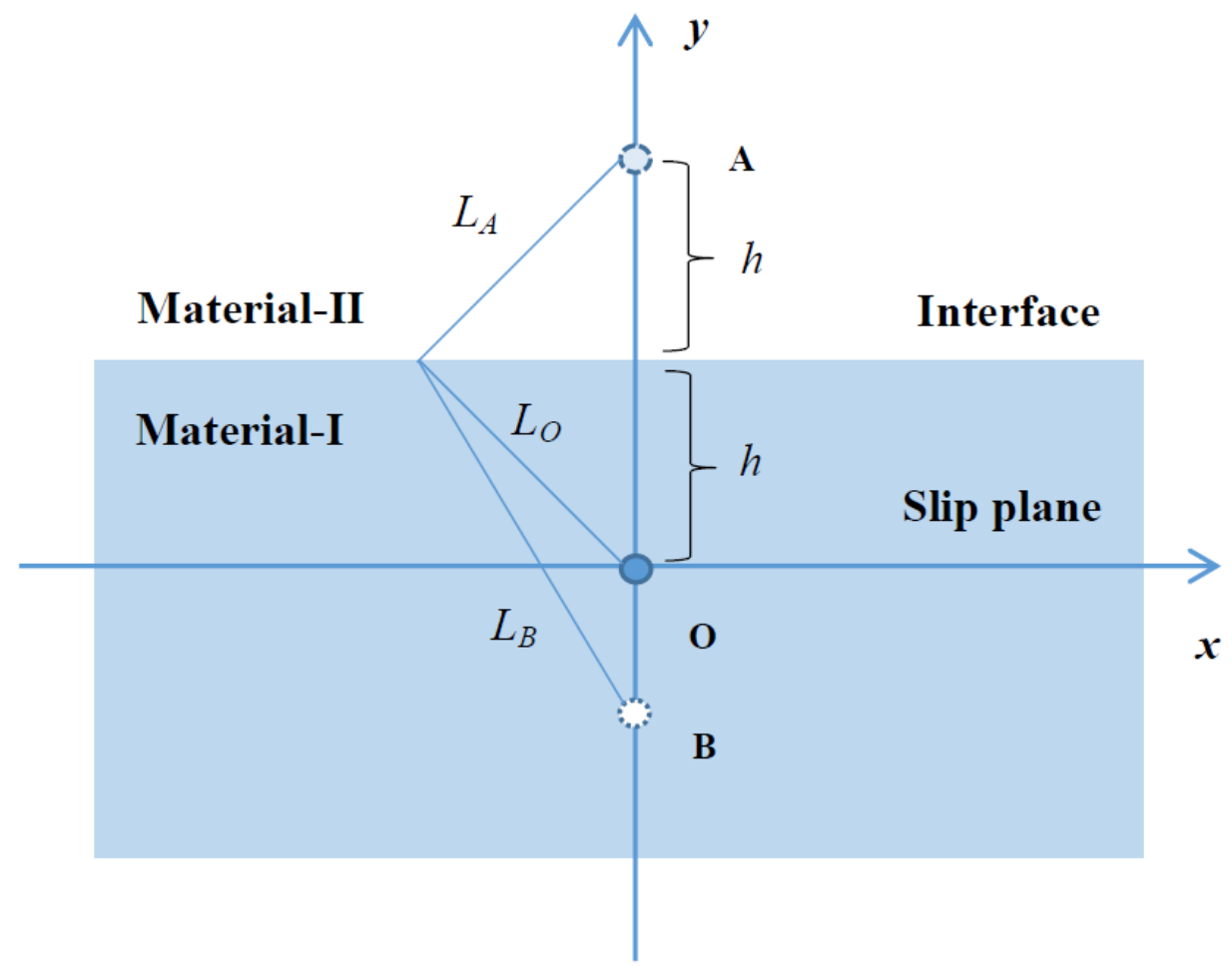

Figure 1. The original dislocation and image dislocations in heterostructure. $h$ is the distance between dislocation and interface. The slip plane is parallel to the interface.

\section{Interfacial Effects on Dislocation Core and Peierls Stress}

Foreman introduced an effective method (Foreman method) to solve the original P-N dislocation equation with nonsinusoidial restoring force [16]. In this paper, we take the Foreman method to solve 
the screw dislocation with the interfacial effects. The typical Foreman solution for the screw dislocation is given as follows:

$$
u(x)=\frac{b}{\pi}\left[1-(\xi-1) \frac{\partial}{\partial \xi}\right] \arctan \frac{p}{\xi},
$$

with $p=2 x / d$ and $d$ the spacing distance of the planes parallel to the slip plane. The core structure parameter $\xi$ is crucially determined by the maximum restoring force. $u(x)(\xi=1)$ reduces to the solution of the original P-N equation with $\sigma_{\max }=\frac{\mu b}{2 \pi d}$ and the core width $\zeta=d / 2$. The core width is determined by $\rho(\zeta)=\frac{1}{2} \rho(0)$. However, the core width will be changed, while the unstable stacking fault energy and interface effects are taken into account. The Foreman method is a modification of the classical P-N solution for the classical P-N dislocation equation. The core structure parameter $\xi$ is introduced into the classical P-N solution to obtain the Foreman solution, Equation (19). It is clear that the classical P-N solution can be obtained from Equation (19) if the core structure parameter $\xi=1$. Furthermore, the Foreman solution is introduced a parametric derivative form. This is helpful in solving the governing dislocation equation, Equation (14).

Substituting the Foreman solution to the dislocation equation and letting $p=\cot \frac{\theta}{2}$, the stress and displacement can be expressed as the parametric forms

$$
\begin{gathered}
\sigma(\theta)=\frac{\mu \kappa b}{2 \pi}\left\{\frac{\cot \frac{\theta}{2}\left[1+\xi^{2}(-1+2 \xi) \cot ^{2} \frac{\theta}{2}\right]}{\left(1+\xi^{2} \cot ^{2} \frac{\theta}{2}\right)^{2}}+\gamma \frac{\cot \frac{\theta}{2}\left[(1+2 \kappa h)^{2}+\xi^{2}(-1+2 \xi) \cot ^{2} \frac{\theta}{2}\right]}{\left[(1+2 \kappa h)^{2}+\xi^{2} \cot ^{2} \frac{\theta}{2}\right]^{2}}\right\} \\
u(\theta)=\frac{b}{\pi}\left(\frac{\pi}{2}-\frac{\theta}{2}+\frac{\xi-1}{2 \xi} \sin \theta\right)
\end{gathered}
$$

with $\kappa=\frac{2}{\xi d}$ and $\theta$ in the range from 0 to $2 \pi$. The core structure parameter $\xi$ can be determined by fitting Equation (20) for the maximum restoring force. It is interesting to find that $\sigma(\theta)=\frac{\mu b}{2 \pi d} \sin \theta$ when $\xi=1$ and $\gamma=0$.

The variations of $\xi$ with respect to distance $h$ for different interface types $\gamma=-1,-0.5,0,0.5,1$ are shown in Figure 2. For comparison, the results of $\sigma_{\max }=\mu b / 8 \pi d$ and $\mu b / 2 \pi d$ are displayed. It worth noting that $\xi$ decreases with $h$ for $\gamma>0$ and increases with $h$ for $\gamma<0$ when $\sigma_{\max }=\mu b / 8 \pi d$. The larger the value of $h$, the fewer influence the interface has on the dislocation core. $\xi$ tends towards the results of $\gamma=0$. The surface and interface effects on core structure become small for large $h$. For the same $h, \gamma>0$ leads to a larger $\xi$ (wider dislocation core) than $\gamma<0$. Namely, a rigid material will extend the dislocation core in a soft material. However, a soft material will compress the dislocation core in a rigid material. In other words, the image dislocation has the same (different) chirality with the original dislocation for $\gamma>0(\gamma<0)$. This means that dislocations at the parallel slip planes with same chirality lead to a wide core, and different chirality lead to a narrow core. It should be kept in mind that the above discussions are based on a fixed maximum restoring force for different $h$. The core structure parameter $\xi$ is near the same for $\sigma_{\max }=\mu b / 2 \pi d$ for different $h$ and $\gamma$ (see Figure 2). Figure 3 a illustrates that the core structure parameter $\xi$ decreases with increasing maximum restoring force $\sigma_{\max }$ for $h=4 d$. It is clear that $\xi>1$ and $\xi<1$ for $\sigma_{\max }<\mu b / 2 \pi d$ and $\sigma_{\max }>\mu b / 2 \pi d$, respectively. The difference between $\xi$ and $\gamma$ is very small for higher $\sigma_{\max }$.

Generally, the mobility is controlled by the dislocation core width $\zeta$ and the unstable stacking fault energy $\gamma_{u s}$. The following formula can be used to predict the Peierls stress of dislocations:

$$
\sigma_{P}=\frac{2 \pi^{2} \gamma_{u} \zeta}{b^{2}} \exp ^{-\frac{2 \pi \zeta}{b}} .
$$



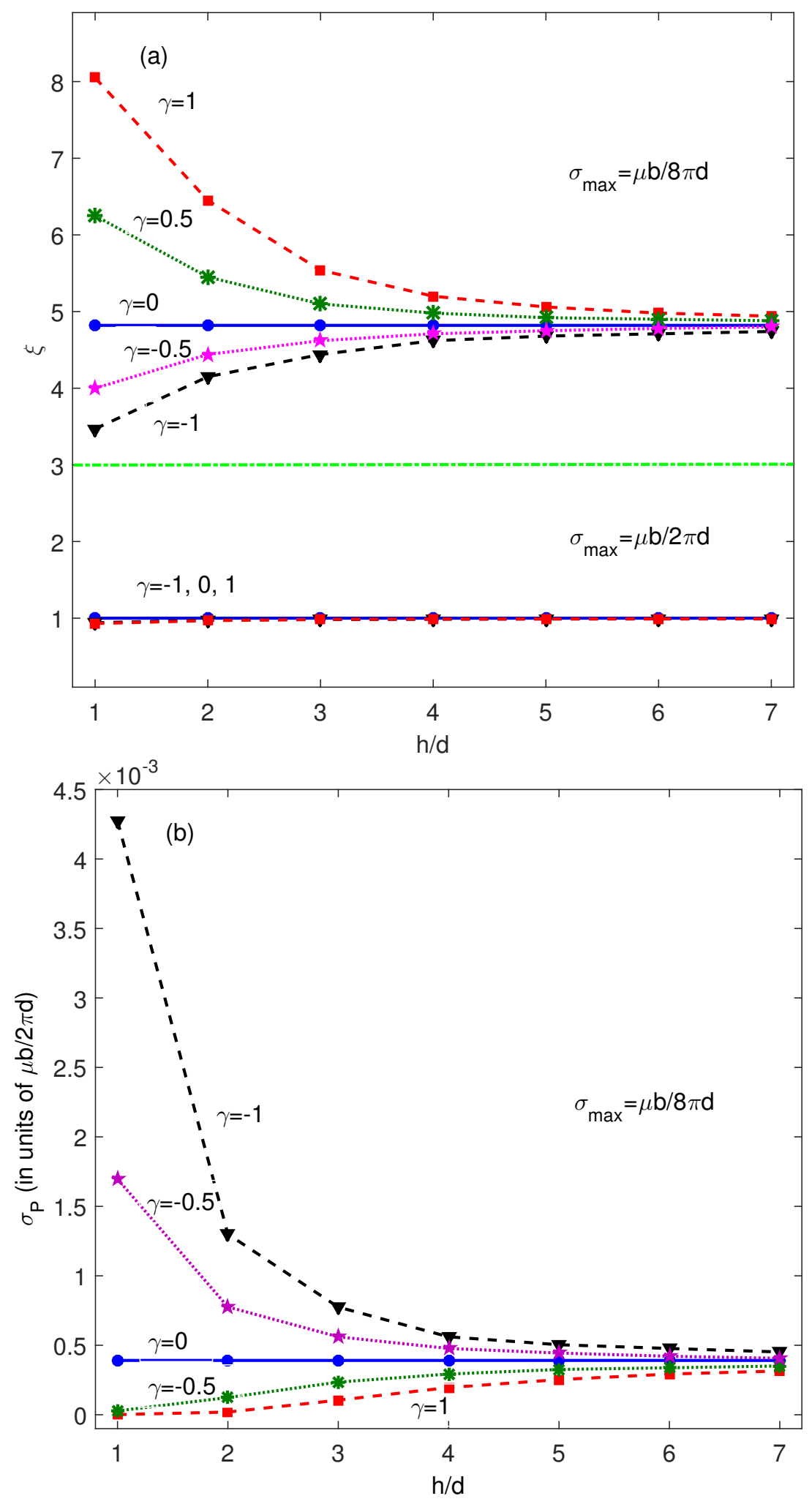

Figure 2. The core structure parameters $\xi$ vary with the distance between dislocation (a) and interface $h(\mathbf{b})$.

Peierls stress for $\sigma_{\max }=\frac{\mu b}{8 \pi d}$ and different interface types are illustrated in Figure $2 \mathrm{~b}$. It is easy to see that Peierls stress can be enhanced by the interface for $\gamma<0\left(\mu_{I I}<\mu_{I}\right)$, and reduced by the interface for $\gamma>0\left(\mu_{I I}>\mu_{I}\right)$. The Peierls stress of screw dislocations in bulk material-I is $3.91 \times 10^{-4} \sigma_{\max }$. We take $h=2 d$ as an example. When $\gamma=-1\left(\mu_{I I}=0\right)$, the Peierls stress of screw 
dislocations is $1.3 \times 10^{-3} \sigma_{\max }$. The Peierls stress is enhanced by the surface by about three times. When $\gamma=-0.5\left(\mu_{I I}=\frac{1}{3} \mu_{I}\right)$, the Peierls stress of screw dislocations is $7.76 \times 10^{-4} \sigma_{\max }$. The Peierls stress decreases with the increasing $\gamma$. The Peierls stress of screw dislocations is $1.24 \times 10^{-4} \sigma_{\max }$ for $\gamma=0.5\left(\mu_{I I}=3 \mu_{I}\right)$. The Peierls stress is reduced by the interface. Furthermore, the interface effect decreases with increasing $h$ and $\sigma_{\max }$ for both $\gamma>0$ and $\gamma<0$. This can be easily understood using Figure 4 . When $h<\zeta$, the original core region will superpose with the image core region. The original dislocation core will be affected by the image core. The surface and interface effects cannot be ignored. The original core width decreases with increasing $\sigma_{\max }$. The distance between the original core region and the image core region become large, and the superposition of core regions becomes weak. The surface and interface effects on the original core are also reduced. Figure 4 also shows that the superposition decreases with increasing $h$.

Based on the above discussions, the conclusion can be made that the interface effects on the core width and Peierls stress are determined by the maximum restoring force $\sigma_{\max }$ (the unstable stacking fault energy $\gamma_{u s}$ ), the distance to interface $h$, and the interface type $\gamma$. In order to elucidate their contributions, we take $h=4 d$ as an example. The Peierls stress has the same order for $\gamma=-1, \gamma=0$, and $\gamma=1$. This is also true for $h=d$, although the Peierls stress has the biggest difference for $\gamma=-1$, $\gamma=0$, and $\gamma=1$. The Peierls stress also has the same order when $h$ in the range of $1 d \sim 7 d$. However, the Peierls stress for $\sigma_{\max }=\frac{\mu b}{2 \pi d}$ is 1000 times that for $\sigma_{\max }=\frac{\mu b}{8 \pi d}$ (see Figure 3). However, the Peierls stress for $\sigma_{\max }=\frac{\mu b}{2 \pi d}$ is only 1.3 times of that for $\sigma_{\max }=\frac{3 \mu b}{8 \pi d}$. The Peierls stress for $\sigma_{\max }=\frac{3 \mu b}{8 \pi d}$ is about 21 times that for $\sigma_{\max }=\frac{\mu b}{8 \pi d}$. Therefore, the interface effect on dislocation will be mainly controlled by the difference between the unstable stacking fault energy (the maximum restoring force) with and without interface. Furthermore, the interface effect is clearer when the unstable stacking energy in bulk material-I is smaller than $\frac{\mu b}{2 \pi d}$.

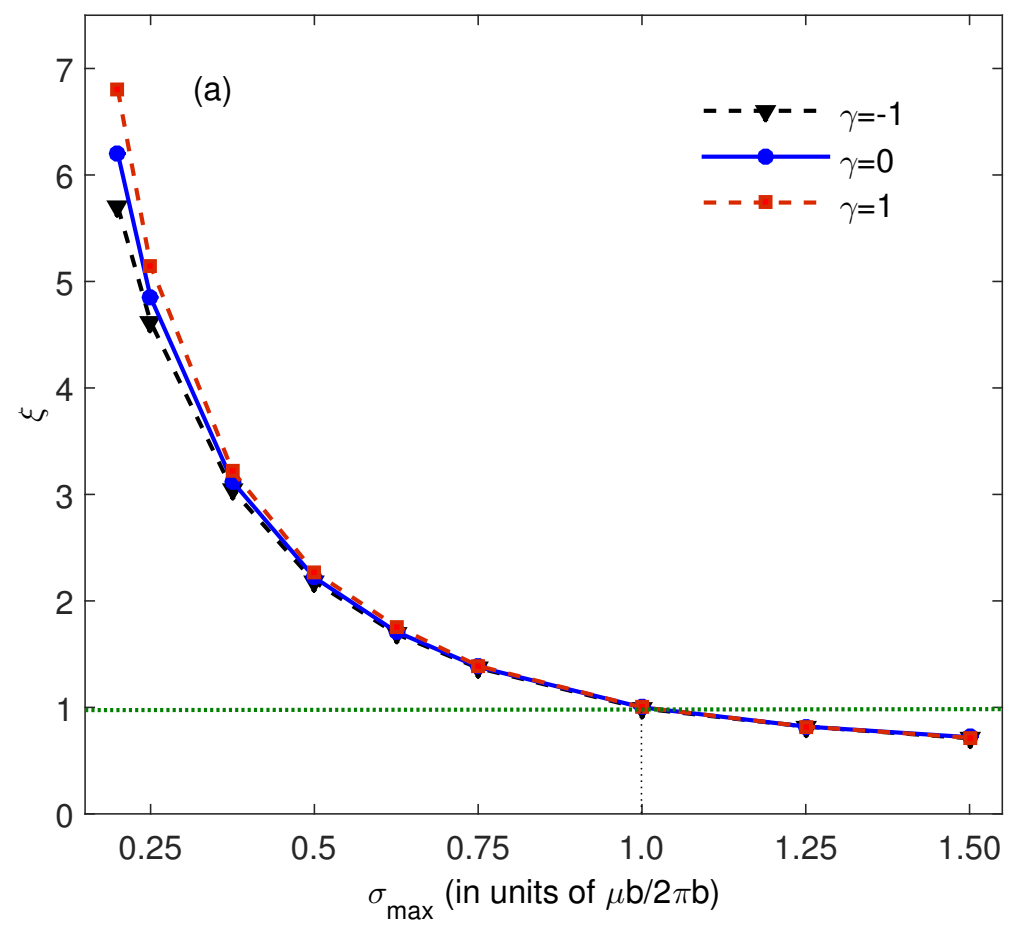

Figure 3. Cont. 


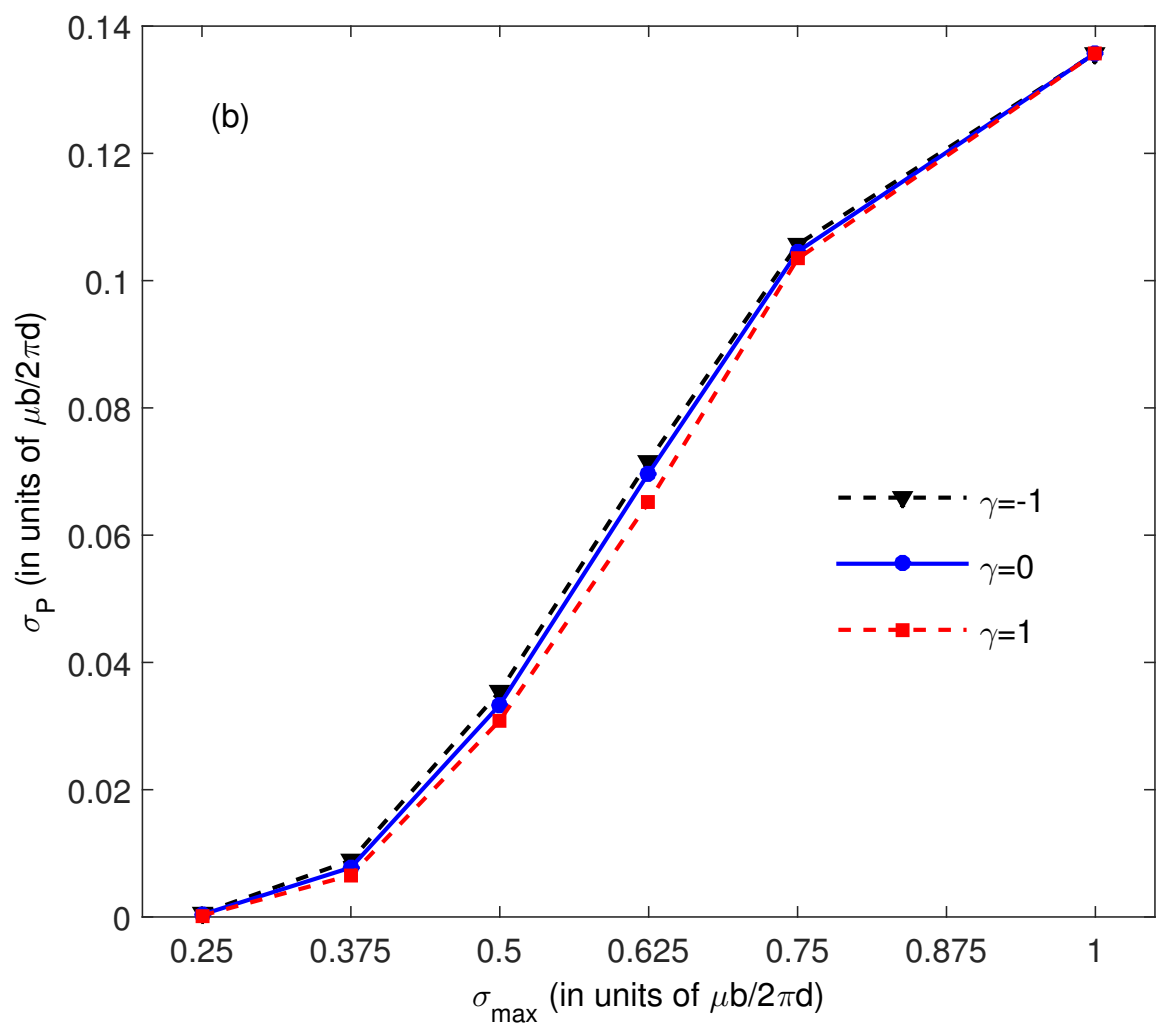

Figure 3. The maximum restoring force $\sigma_{\max }$ on the core structure parameter $\xi$ (a) and Peierls stress $\sigma_{P}$ (b) (take $h=4 d$ as an example).

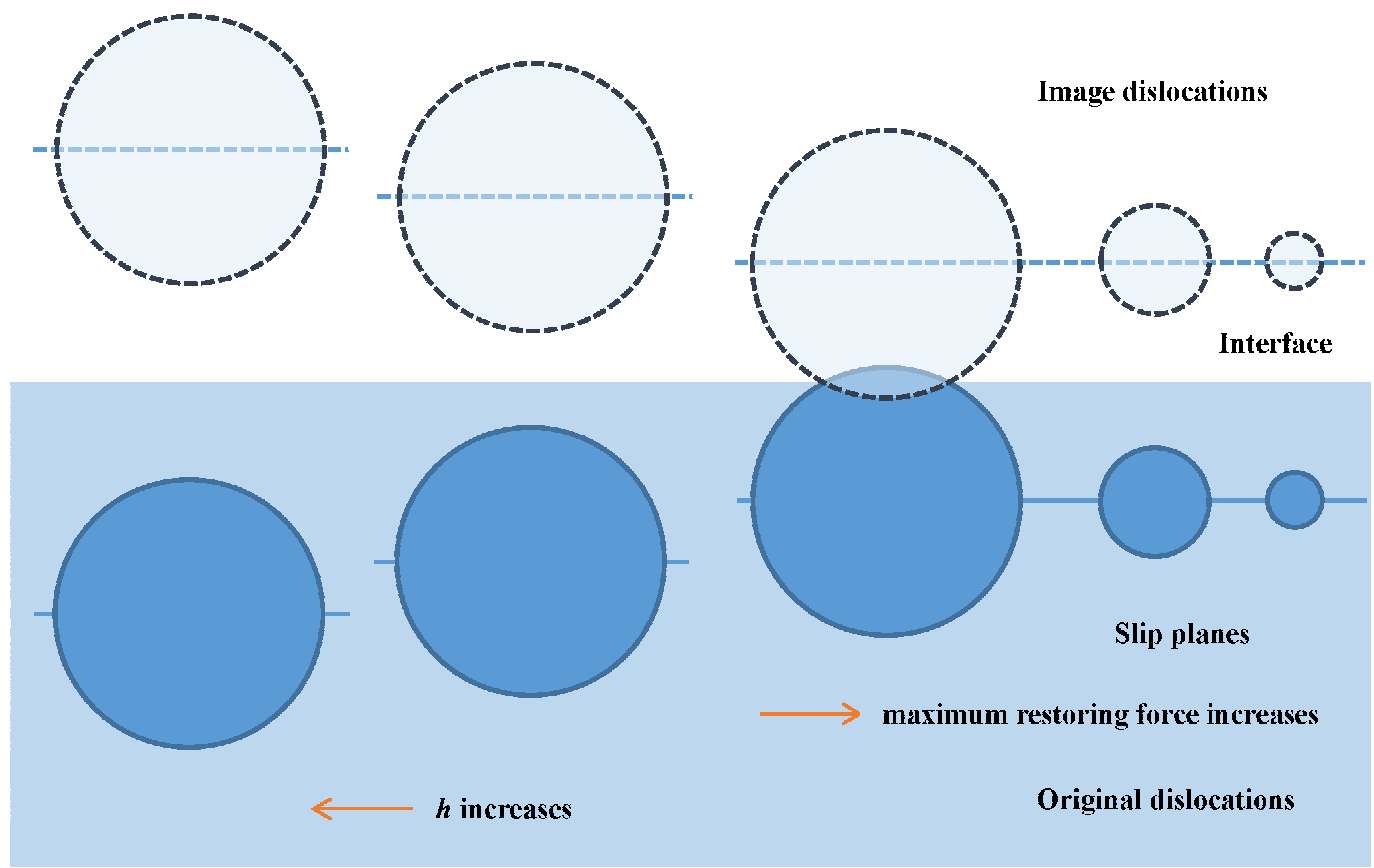

Figure 4. Image dislocations core structures for different maximum restoring force $\sigma_{\max }$ and the distance between dislocation and interface $h$. Larger $\sigma_{\max }$ leads to narrower dislocation core, and the superposition between original core and image core will become smaller. $h$ has the same effects. 


\section{Screw Dislocations in Al/TiC Heterostructure}

Generally, material-I and material-II have different lattice constants and shear moduli. The atoms near the interface adjust their position to reduce the elastic strain energy. These will change the unstable stacking fault energy in material-I and material-II. Compared to interfacial type $(\gamma)$ and distance $(h)$, the contributions of maximum restoring force (unstable stacking fault energy) needs to be discussed further. In order to investigate the direct effect of interface, we calculated the unstable stacking fault energy for $\mathrm{Al} / \mathrm{TiC}$ heterostructures as a model interface using first principles methods. In order to make the paper easy to read, the detailed computation procedures are not listed (for detailed computation procedures, refer to [17]). The interface is (100) plane, and the Burgers vector of screw dislocation is along $\langle 110\rangle$ directions. The calculated lattice constants of $\mathrm{Al}$ and $\mathrm{TiC}$ are 4.04 and 4.33, respectively. The shear modulus is $26.0 \mathrm{GPa}$ for $\mathrm{Al}$ and $164.5 \mathrm{GPa}$ for TiC. According to the large difference in shear modulus between $\mathrm{Al}$ and $\mathrm{TiC}$, we can use the coherent interface approximation. The softer $\mathrm{Al}$ is stretched to match the dimension of TiC. The axis normal to the interface is still the dimension of Al. If the difference of shear modulus is small, the material with larger constants will be stretched, and the other material will be compressed.

The unstable stacking fault energy is listed in Table 1. The unstable stacking fault energy for bulk $\mathrm{Al}$ is $0.837 \mathrm{~J} / \mathrm{m}^{2}$. When $\mathrm{Al}$ is stretched, the unstable stacking fault (USF) energy is $0.527 \mathrm{~J} / \mathrm{m}^{2}$. The stretch reduces the USF energy by $0.31 \mathrm{~J} / \mathrm{m}^{2}$. When the $\mathrm{Al} / \mathrm{TiC}$ interface is formed, the unstable stacking fault energies in $\mathrm{Al}$ are $0.396,0.540,0.545$, and $0.475 \mathrm{~J} / \mathrm{m}^{2}$ for distances $h=1 d, 2 d, 3 d$, and $4 d$, respectively. The changes of energy between stretched $\mathrm{Al}$ and $\mathrm{Al}$ in the interface are $0.131,-0.013$, -0.018 , and $0.052 \mathrm{~J} / \mathrm{m}^{2}$, respectively. The USF energy for bulk TiC is $3.582 \mathrm{~J} / \mathrm{m}^{2}$. In the coherent interface approximation, $\mathrm{TiC}$ is not stretched. After the $\mathrm{Al} / \mathrm{TiC}$ interface is formed, the unstable stacking fault energies in TiC are 3.650, 3.568, 3.576, and $3.582 \mathrm{~J} / \mathrm{m}^{2}$. The energy differences are -0.068 , $0.014,0.006$, and $0 \mathrm{~J} / \mathrm{m}^{2}$. The interface formation can be postulated including two steps : (1) stretch soft $\mathrm{Al}$, (2) form $\mathrm{Al} / \mathrm{TiC}$ interface by $\mathrm{TiC}$ and stretched $\mathrm{Al}$. The above calculation results show that step (1) provides the main contribution to the unstable stacking fault energy in comparison with step (2). Therefore, the interface effects on USF stacking are originated from the stretching of Al.

The change of energy between $h=1 d$ and stretched $\mathrm{Al}$ is larger than $h=2 d, 3 d$, and $4 d$. The calculated interfacial USF energy is $1.397 \mathrm{~J} / \mathrm{m}^{2}$, which is larger than bulk $\mathrm{Al}$ and stretched $\mathrm{Al}$. This means that $\mathrm{Al}$ and $\mathrm{C}$ atoms at the interface have strong covalent bonding, resulting in a smaller unstable stacking fault energy for $h=1 d$. However, the interfacial bonds have little impact on $h=2 d$, $3 d$, and $4 d$, due to the locality of stacking fault energy.

For screw dislocations in $\mathrm{Al}$ and $\mathrm{TiC}$, the interface types $(\gamma)$ are 0.727 (rigid interface) and -0.727 (soft interface), respectively. The core width and Peierls stress is listed in Table 1. The Peierls stress of screw dislocations in interface- $\mathrm{Al}$ is smaller than that in bulk $\mathrm{Al}$ due to the stretching when forming the $\mathrm{Al} / \mathrm{TiC}$ interface. The lattice constants are unaltered when forming the $\mathrm{Al} / \mathrm{TiC}$ interface due to large shear modulus. The Peierls stress is nearly the same in bulk TiC and interface-TiC.

Table 1. The unstable stacking fault energy $\gamma_{u s}$ (in units of $\mathrm{J} / \mathrm{m}^{2}$ ), the maximum restoring force $\sigma_{\max }$ (in units of GPa), the half width of dislocation $\zeta$ (in units of Burgers vector), and the Peierls stress $\sigma_{P}$ (in units of GPa).

\begin{tabular}{|c|c|c|c|c|c|c|c|c|}
\hline & \multicolumn{4}{|c|}{ Al } & \multicolumn{4}{|c|}{$\mathrm{TiC}$} \\
\hline & $\gamma_{u s}$ & $\sigma_{\max }$ & $\zeta$ & $\sigma_{p}$ & $\gamma_{u s}$ & $\sigma_{\max }$ & $\zeta$ & $\sigma_{p}$ \\
\hline Bulk & 0.837 & 9.20 & 0.225 & 3.16 & 3.582 & 36.75 & 0.356 & 8.77 \\
\hline $\mathrm{Bulk}_{s}$ & 0.527 & 5.80 & 0.357 & 1.38 & - & - & - & - \\
\hline $1 d$ & 0.396 & 4.35 & 0.476 & 0.65 & 3.650 & 37.45 & 0.350 & 9.15 \\
\hline $2 d$ & 0.540 & 5.94 & 0.348 & 1.46 & 3.568 & 36.61 & 0.358 & 8.70 \\
\hline $3 d$ & 0.545 & 5.99 & 0.345 & 1.48 & 3.576 & 36.69 & 0.357 & 8.74 \\
\hline $4 d$ & 0.475 & 5.22 & 0.396 & 1.08 & 3.582 & 36.75 & 0.356 & 8.77 \\
\hline
\end{tabular}




\section{Conclusions}

Due to the equivalence of dislocations and point charges in elastic strain field and electrostatics, the image method is used to describe the interface effects on the dislocation elastic strain field. The interface effects on dislocations are represented by image dislocations. The governing equation for screw dislocation in heterostructure is constructed using the image dislocation method. The interface type $(\gamma)$ and distance $(h)$ effects are contained in the equation. The P-N dislocation equations in bulk and in semi-infinite materials can be recovered when $\gamma=0$ and $\gamma=-1$, respectively. The Peierls stress can be enhanced by a soft interface and decreased by a hard interface. The hard and soft interfaces can be described by $\gamma<0$ and $\gamma>0$. With the increasing of $h$, the effects on dislocations of the interface become small. In order to investigate the interface on the unstable stacking fault energy and dislocation properties, the $\mathrm{Al} / \mathrm{TiC}$ heterostructures were studied as a model interface. It was found that the changes of the unstable stacking fault energy mainly originated from the mismatch of lattice constants and shear modulus at the interface. Then, the changes of the unstable stacking fault energy also have important effects on dislocation properties comparing with $\gamma$ and $h$.

Acknowledgments: Project Supported by Science Challenge No.TZ2016003 and Project Supported by the Fundamental Research Funds for the Central Universities No.2017CDJQJ308822, Project Supported by the Natural Science Foundation of China (91634106).

Author Contributions: J.W. and X.W. conceived the paper; T.S. and W.X. performed the first principle calculations; X.W. and R.W. analyzed the results; J.W. wrote the paper.

Conflicts of Interest: We declare that we have no conflict of interest.

\section{References}

1. Hirth, J.P.; Lothe, J. Theory of Dislocations, 2nd ed.; Wiley: New York, NY, USA, 1982.

2. Peierls, R. The size of a dislocation. Proc. Phys. Soc. 1940, 52, 34.

3. Nabarro, F.R.N. Dislocations in a simple cubic lattice. Proc. Phys. Soc. 1947, 59, 256.

4. Vitek, V. Intrinsic stacking faults in body-centred cubic crystals. Philos. Mag. 1968, 18, 773-786.

5. Joos, B.; Ren, Q.; Duesbery, M.S. Peierls-Nabarro model of dislocations in silicon with generalized stacking-fault restoring forces. Phys. Rev. B 1994, 50, 5890.

6. Pei, Z.R.; Ma, D.C.; Friak, M.; Svendsen, B.; Raabe, D.; Neugebouer, J. From generalized stacking fault energies to dislocation properties: Five-energy-point approach and solid solution effects in magnesium. Phys. Rev. B 2015, 92, 064107.

7. Gouriet, K.; Carrez, P.; Cordier, P.; Guitton, A.; Joulain, A.; Thilly, L.; Tromas, C. Dislocation modelling in Ti2AlN MAX phase based on the Peierls-Nabarro model. Philos. Mag. 2015, 95, 2539-2552.

8. Chen, C.; Meng, F.C.; Sun, J. Effects of Mg and Al doping on dislocation slips in GaN. J. Appl. Phys. 2016, $119,064302$.

9. Xiao, Z.M.; Chen, B.J. A screw dislocation interaction with a coated fiber. Mech. Mater. 2000, 32, 485-494.

10. Lee, C.L.; Li, S. The size effect of thin films on the Peierls stress of edge dislocations. Math. Mech. Solids 2008, 13, 316-335.

11. Gars, B.; Markenscoff, X. The Peierls stress for coupled dislocation partials near a free surface. Philos. Mag. 2012, 92, 1390-1421.

12. Cheng, X.; Shen, Y.; Zhang, L.; Liu, X.H. Surface effect on the screw dislocation mobility over the Peierls barrier. Philos. Mag. Lett. 2012, 92, 270-277.

13. Head, A.K. The Interaction of Dislocations and Boundaries. Philos. Mag. 1953, 44, 92-94.

14. Yao, Y.; Wang, T.C.; Wang, C.Y. Peierls-Nabarro model of interfacial misfit dislocation: An analytic solution. Phys. Rev. B 1999, 59, 8232.

15. Rice, J.R. Dislocation nucleation from a crack tip: An analysis based on the Peierls concept. J. Mech. Phys. Solids 1992, 40, 239-271. 
16. Foreman, A.J.; Jaswon, M.A.; Wood, J.K. Factors Controlling Dislocation Widths. Proc. Phys. Soc. A 1951, 64, 156.

17. Wu, X.Z.; Sun, T.; Wang, R.; Liu, L.L.; Liu, Q. Energy investigations on the adhesive properties of $\mathrm{Al} / \mathrm{TiC}$ interfaces: First-principles study. Physica B 2014, 449, 269-273.

(C) 2018 by the authors. Licensee MDPI, Basel, Switzerland. This article is an open access article distributed under the terms and conditions of the Creative Commons Attribution (CC BY) license (http:/ / creativecommons.org/licenses/by/4.0/). 